\title{
Stress concentrations in keyways and optimization of keyway design
}

\author{
Pedersen, Niels Leergaard
}

Published in:

Journal of Strain Analysis for Engineering Design

Link to article, DOI:

10.1243/03093247JSA632

Publication date:

2010

Document Version

Publisher's PDF, also known as Version of record

Link back to DTU Orbit

Citation (APA):

Pedersen, N. L. (2010). Stress concentrations in keyways and optimization of keyway design. Journal of Strain Analysis for Engineering Design, 45(8), 593-604. https://doi.org/10.1243/03093247JSA632

\section{General rights}

Copyright and moral rights for the publications made accessible in the public portal are retained by the authors and/or other copyright owners and it is a condition of accessing publications that users recognise and abide by the legal requirements associated with these rights.

- Users may download and print one copy of any publication from the public portal for the purpose of private study or research.

- You may not further distribute the material or use it for any profit-making activity or commercial gain

- You may freely distribute the URL identifying the publication in the public portal

If you believe that this document breaches copyright please contact us providing details, and we will remove access to the work immediately and investigate your claim 


\title{
Stress concentrations in keyways and optimization of keyway design
}

\author{
N L Pedersen \\ Department of Mechanical Engineering, Solid Mechanics, Technical University of Denmark, Lyngby, Denmark
}

The manuscript was received on 23 December 2009 and was accepted after revision for publication on 22 April 2010.

DOI: 10.1243/03093247JSA632

\begin{abstract}
Keys and keyways are one of the most common shaft-hub connections. Despite this fact very little numerical analysis has been reported. The design is often regulated by standards that are almost half a century old, and most results reported in the literature are based on experimental photoelastic analysis. The present paper shows how numerical finite element (FE) analysis can improve the prediction of stress concentration in the keyway. Using shape optimization and the simple super elliptical shape, it is shown that the fatigue life of a keyway can be greatly improved with up to a 50 per cent reduction in the maximum stress level. The design changes are simple and therefore practical to realize with only two active design parameters.
\end{abstract}

Keywords: Keyway, parallel key, stress concentration, optimization, Laplace equation, FE

\section{INTRODUCTION}

Keys and keyways commonly connect shaft and hubs. The designs of these are controlled by different standards, e.g. reference [1]. Different design principles are possible; these include parallel keys, tapered keys, or Woodruff keys, see e.g. references [2] and [3]. Among these, the most common is the parallel key, which is the subject of the present paper. The key and keyway design is fully controlled by the standards based on only one parameter - the shaft diameter. It is remarkable that very little effort has been made to improve the design with respect to fatigue, i.e. by minimizing the stress concentrations. This has already been pointed out by Orthwein [4] and, to the current author's knowledge, very little has been done since. Other designs are possible and have been proposed in the literature, e.g. references [5] and [6].

The first paper addressing the torsional stiffness of shafts with a kind of keyway is probably that of Filon [7]. In this paper, the shafts were modelled with elliptical cross-section and the keyways were modelled as hyperbolae. Following this analytical paper, there have been a number of experimental papers dealing with the stress concentrations of key and keyway connections. Many of these papers have used photoelastic analysis, see e.g. references [8] to [13]. Other papers have used electroplating of copper to the surface, see e.g. references [14] and [15]. In addition to references [7] and [8], other papers have dealt with experimental stress concentration verification, see the references in Orthwein [4].

The most commonly used reference with respect to stress concentration factors is Peterson [16], which is reproduced and extended in Pilkey [17]. The keyway results reported here are taken from the references $[\mathbf{8}, \mathbf{9}]$ and $[\mathbf{1 5}]$. The use of finite element (FE) modelling and computational power makes it possible to improve these results, but it seems that this has not yet been done.

The purpose of the present paper is therefore twofold; first find stress concentration by using FE analysis of existing standard designs, and second improve/optimize the keyway design by lowering the stress concentration. The keyway related stress is indeed fully three dimensional as also stated in Peterson [18]. A number of different factors will have an influence on the needed FE analysis complexity and on the resulting maximum stresses found by the analyses. These factors are:

(a) loading: tension, bending, or torsion;

(b) key: loaded with or without the key inserted in the keyway;

(c) stress: at the keyway end or in the prismatic part. 


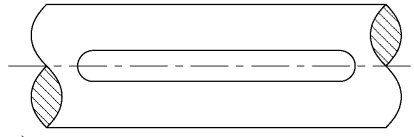

a)

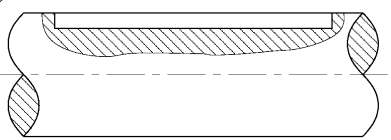

b)
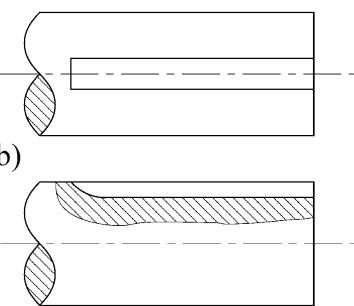

Fig. 1 The two standard keyway ends for parallel keys. (a) end-milled or profile keyway; (b) sledrunner keyway

Restricting the numerical analysis, the present paper deals only with torsion; with respect to the other loads or any load combinations the reader is referred to Fessler et al. [9]. To make an easy comparison with the numerical and experimental work in Leven [8] possible, the keyway is loaded in torsion without the key. This means that there is no need for contact analysis, which would complicate the numerical analysis considerably. The reported results in Okubo et al. [15] state that there is a difference in the maximum stress for pure torsional loading without the key relative to torsion applied through the key. The experiments presented in reference [15] were in two groups (group A and B) for the different relative sizes of the keyway to the shaft diameter. The reported experimental result is that in the prismatic keyway part the maximum stress is $8-12$ per cent for group A and 4-7 per cent for group $B$ greater with a key relative to no key, while the difference is $16-24$ per cent for group A and 12-14 per cent for group B at the key end. These values were relatively unaffected by different ratios of fillet radius to shaft diameter. This leads to the conclusion that the true stress concentrations can be found from a study without the torsion coming from the key by adding a maximum 12 per cent to the stresses in the prismatic part.

The end of a keyway has two standard designs, shown in Fig. 1. The profile keyway is cut by an endmill while the sled-runner keyway is cut by an ordinary milling cutter. The stress concentrations at the keyway end are most severe for the profile keyway, so with respect to fatigue the sled-runner is the best design. Orthwein [4] suggested a design change to the sled-runner keyway end that further improves the fatigue properties. Leven [8] found the stress concentration factor for pure torsion for a profile keyway end to be $K_{\mathrm{t}}=3.4$ for a width of keyway to diameter ratio equal to $b / d=1 / 4$. This value was unaffected by the keyway bottom fillet radius. If the profile keyway end design is to be improved we should move away from the circular design; this would most probably increase the machining cost and is not discussed further in this paper. For the sled-runner keyway in pure torsion the stress concentration factor is higher in the keyways prismatic part relative to the keyway end if the same milling cutter is used for the hole cutting operation.

With the simplification made the analysed stress concentration factor in the present paper is fully controlled by the keyway fillet in the bottom of the prismatic part. The design domain is two dimensional and shown in Fig. 2.

Obeying the standards, the only way to improve the stress concentrations for the design in Fig. 2 is to select the maximum fillet radius $r$. Previous work on shape optimization in relation to machine elements, see references [19] and [20], has shown that changing from the circular shape to an elliptical shape significantly affects the stress concentrations. This is also demonstrated in the present paper.

The current paper is organized as follows. In section 2 the torsional problem is formulated mathematically and the FE implementation is presented. Section 3 presents the results for standard designs, and a practical curve-fitted equation for the stress concentration based on the ratio values $r / d, t / d$ and $b / d$ is given. The design optimization is presented in section 4 where different modifications to the standard design are proposed, resulting in large reductions in the stress concentrations. This leads to the proposed new standard keyway design in section 5 .

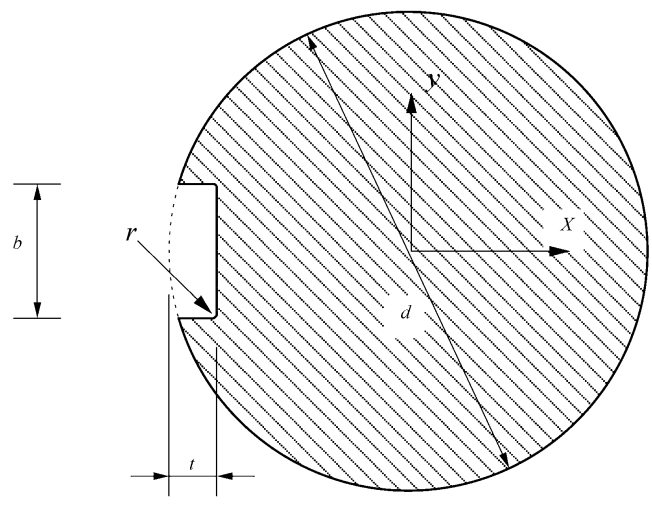

Fig. 2 Cross-section of prismatic part of parallel keyway, the coordinate system is placed at the shaft axis. The relative dimensions correspond to a $d=100 \mathrm{~mm}$ shaft according to DIN 6885-1 [1], $(t=10 \mathrm{~mm}, b=28 \mathrm{~mm}, 0.4 \mathrm{~mm} \leqslant r \leqslant 0.6 \mathrm{~mm})$

\section{MATHEMATICAL FORMULATION AND FE}

The torsional moment is given by

$$
M_{\mathrm{t}}=G J \frac{\phi}{l}
$$


where $G$ is the shear modulus of elasticity, $J$ is the cross-sectional torsional stiffness factor, $\phi$ is the angular rotation of torsional cross-section, and $l$ is the shaft length. In the literature it is common to use $\theta=\phi / l$, i.e. angular rotation per length. It is assumed that a prismatic shaft is aligned with a Cartesian coordinate system with the $x$-, $y$-, and $z$-directions such that the shaft axis is aligned with the $z$ direction. Saint-Venant have introduced the warping function $\Psi(x, y)$ by which the shaft displacement under torsion is given by

$$
v_{x}=-y z \frac{\phi}{l} \quad v_{y}=x z \frac{\phi}{l} \quad v_{z}=\Psi(x, y) \frac{\phi}{l}
$$

Using this definition the cross-section shear stresses (all other stresses are zero) are given by

$$
\tau_{z x}=\tau_{x z}=\left(\frac{\mathrm{d} \Psi}{\mathrm{d} x}-y\right) G \frac{\phi}{l} \quad \tau_{z y}=\tau_{y z}=\left(\frac{\mathrm{d} \Psi}{\mathrm{d} y}+x\right) G \frac{\phi}{l}
$$

With zero volume force the force equilibrium gives the Laplace differential equation that the warping function must fulfil

$$
\Delta \Psi=0
$$

To solve this differential equation the boundary conditions are needed. There is no surface traction for free boundaries. If the normal to the surface is defined as $\left\{n_{x}, n_{y}\right\}^{T}$ then the condition of no surface traction is given by

$$
\left\{n_{x}, n_{y}\right\}\left\{\begin{array}{l}
\tau_{z x} \\
\tau_{z y}
\end{array}\right\}=0
$$

This can be reformulated into a Neumann boundary condition for the warping function by using equation (3)

$$
\left\{n_{x}, n_{y}\right\}\left\{\begin{array}{l}
\frac{\mathrm{d} \Psi}{\mathrm{d} x} \\
\frac{\mathrm{d} \Psi}{\mathrm{d} y}
\end{array}\right\}=\left\{n_{x}, n_{y}\right\}\left\{\begin{array}{c}
y \\
-x
\end{array}\right\}
$$

It is possible to utilize symmetry, see Fig. 3 where half the cross-section of a shaft is shown. The boundary condition for a symmetry line is given by

$$
\left\{-n_{y}, n_{x}\right\}\left\{\begin{array}{l}
\tau_{z x} \\
\tau_{z y}
\end{array}\right\}=0
$$

If the symmetry line demonstrates that $y=0$, as in Fig. 3, then the boundary condition for the symmetry line (7) can be simplified. Since $n_{x}=0$ and $n_{y}=1$, the boundary condition becomes $\tau_{z x}=0$ or by using equation (3) $d \Psi / d x=0$. This is identical with the Dirichlet boundary condition

$$
\Psi=C
$$

where $C$ is an arbitrary constant. Since only the first derivative of the warping function is of interest, we may select $C=0$. By formulating the torsional problem as equation (4) with the boundary conditions (6) and (8) it is possible to use a standard partial differential equation (PDE) solver. In the present paper the program COMSOL is used [21].

It should be noted that the displacements (2) are all defined relative to a coordinate system placed at the centre of torsion. The calculation of the involved strains and stresses are, however, insensitive to any movement or rotation of the coordinate system.

\subsection{Stress concentration}

The stress concentration is most often defined as

$$
K_{\mathrm{t}}=\frac{\sigma_{\mathrm{max}}}{\sigma_{\mathrm{nom}}}
$$
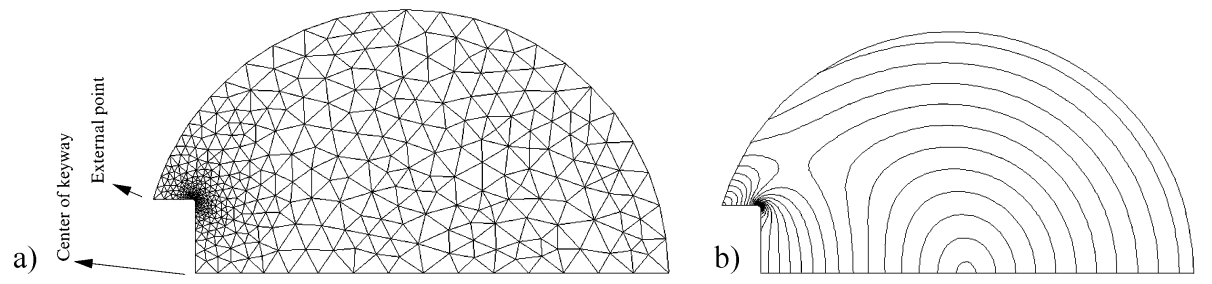

Fig. 3 The figures are for half the shaft given in Fig. 2. (a) Example of a finite element mesh, the illustrated mesh has 917 elements. The Dirichlet boundary condition (8) is applied to the bottom edge while the Neumann boundary condition (6) is applied to the remaining edges. (b) Iso lines of resulting stress level, indicating the stress concentration at the corner 
where $\sigma_{\text {nom }}$ is the nominal stress, i.e. the maximum stress without the keyway and $\sigma_{\max }$ is the maximum stress with the keyway. Both stresses are the greatest principal stress. The subscript $t$ indicates that it is a theoretical stress concentration based only on geometry and loading/boundary condition, no material sensitivity is included. For torsional problems the stress concentration may be given as

$$
K_{\mathrm{ts}}=\frac{\tau_{\max }}{\tau_{\mathrm{nom}}}
$$

where for the present torsional problem

$$
\begin{aligned}
\tau_{\text {nom }} & =\frac{16 M_{\mathrm{t}}}{\pi d^{3}} \\
\tau_{\max } & =\left(\sqrt{\tau_{z x}^{2}+\tau_{z y}^{2}}\right)_{\text {max }}
\end{aligned}
$$

The nominal stress and the maximum stress are found under the same external loading. By the assumption of linear elasticity the external load size does not influence the stress concentration. The size of $M_{\mathrm{t}}$ is selected such that

$$
G \frac{\phi}{l}=1 \mathrm{~N} / \mathrm{m}^{3}
$$

This leads to the nominal stress and maximum stress given as

$$
\begin{aligned}
\tau_{\text {nom }} & =\frac{d}{2} \mathrm{~N} / \mathrm{m}^{3} \\
\tau_{\max } & =\left[\frac{J_{\mathrm{c}}}{J_{\mathrm{k}}} \sqrt{\left(\frac{\mathrm{d} \Psi}{\mathrm{d} x}-y\right)^{2}+\left(\frac{\mathrm{d} \Psi}{\mathrm{d} y}+x\right)^{2}}\right]_{\max } \mathrm{N} / \mathrm{m}^{3}
\end{aligned}
$$

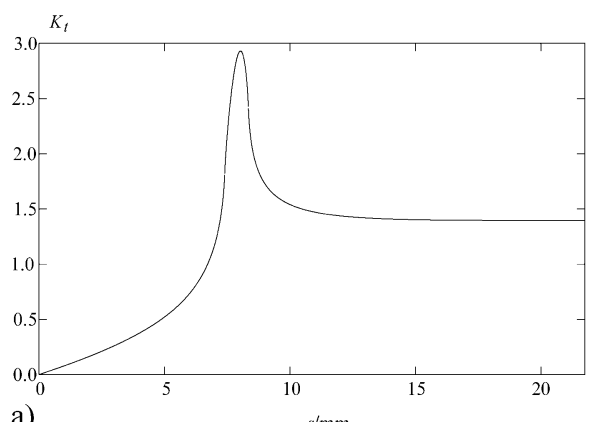

where $J_{\mathrm{c}}$ is the cross-sectional torsional stiffness factor for the circular shaft and $J_{\mathrm{k}}$ is the crosssectional torsional stiffness factor for the shaft with a keyway

$$
\begin{aligned}
& J_{\mathrm{c}}=\frac{\pi d^{4}}{32} \\
& J_{\mathrm{k}}=\int_{A}\left[-\left(\frac{\mathrm{d} \Psi}{\mathrm{d} x}-y\right) y+\left(\frac{\mathrm{d} \Psi}{\mathrm{d} y}+x\right) x\right] \mathrm{d} A
\end{aligned}
$$

\subsection{FE model}

A FE model example is shown in Fig. 3. The shaft design is the DIN standard presented in Fig. 2. Only half the shaft is necessary for the modelling. The bottom edge is a symmetry line so here the Dirichlet boundary condition (8) is applied. The Neumann boundary condition (6) is applied to the remaining edges. The number of elements in the shown mesh is limited (917 elements) for illustrative purposes. The numerical calculations performed in this paper have all been performed with a much higher number of elements (30000 to 60000 ). Convergence tests have been made to confirm the FE results.

The maximum stress is of primary interest, since this stress controls the stress concentration. The maximum stress is in all numerical calculations found at the keyway boundary. In Fig. 4 the stress concentration is shown along the keyway boundary ( $s$ is the arc length), in the close up, Fig. 4(b), the stress concentration along the fillet is shown. From an optimization point of view it is clear that this is not optimal because the stress is expected to be constant along major parts of the surface in order for

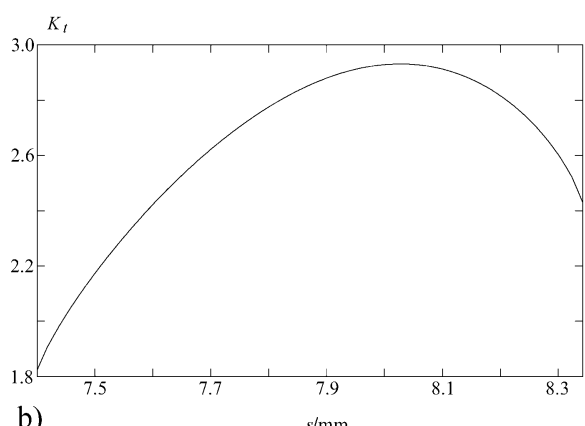

b)

Fig. 4 The figures are for half the shaft shown in Fig. 2 and show the stress concentration as a function of the arc length. (a) The stress concentration factor along the keyway boundary starting from the external point until the centre point. (b) Stress concentration close up, here only shown along the $r=0.6 \mathrm{~mm}$ fillet at the corner. The maximum value is $K_{\mathrm{t}}=2.93$; with a fillet radius of $0.4 \mathrm{~mm}$, which is also allowed by the standard, the value is $K_{\mathrm{t}}=3.32$ 
the design to be optimal, see e.g. reference [19]. The stress level is such that for a fillet radius of $r=0.6 \mathrm{~mm}$ we find $K_{\mathrm{t}}=2.93$; with a fillet radius of $r=0.4 \mathrm{~mm}$, which is also allowed by the standard, the value is $K_{\mathrm{t}}=3.32$. This is a rather large variation in the stress concentration for designs that fulfil the standard geometry.

A fine mesh near the point of stress concentration is needed in order for the FE analysis to return the correct maximum stress value. For the semi-circular fillet designs defined by the standard, see section 3 , the mesh densities are controlled by the FE program. Convergence tests have been made ensure that the mesh density is sufficiently large to ensure reliable results. For the optimized designs in section 4 the outer boundary is discritized such that there are 500 nodes along the fillet. This results in a high accuracy of the reported stress concentration factors.

\section{STRESS CONCENTRATION OF FILLET KEYWAYS (DIN)}

In the standard keyway design [1] the fillet of the prismatic part is within tolerances so that $r / d$ may vary for the same diameter, as seen in Fig. 2. The standard also specifies the ratios $b / d$ and $t / d$ depending on the specific shaft diameter. The diameter range is $6 \mathrm{~mm} \leqslant d \leqslant 500 \mathrm{~mm}$ according to the standard and the limits to the different ratios are

$$
\frac{1}{5} \leqslant \frac{b}{d} \leqslant \frac{5}{12} \quad \frac{31}{500} \leqslant \frac{t}{d} \leqslant \frac{1}{4} \quad \frac{7}{2300} \leqslant \frac{r}{d} \leqslant \frac{16}{600}
$$

The variations of depth ratio $t / d$ and width ratio $b /$ $d$ are shown in Fig. 5(a) and the upper and lower limit for the fillet ratio $r / d$ are shown in Fig. 5(b), all according to DIN 6885.
From Fig. 5 it is clear that there is a large variation in the design. The already published stress concentration for the prismatic part in pure torsion is based on Leven [8] and the results are given for the specific case of $b /$ $d=0.25$ and $t / d=0.125$. It is doubtful that these are suitable average values for the whole range of keyway designs according to DIN 6885. From the stress concentration values found for the $100 \mathrm{~mm}$ shaft in the previous section this seems not to be the case. The results presented in Leven [8] overestimate the $K_{\mathrm{t}}$ values slightly and therefore the curve fit presented in Pilkey [17] is also an overestimation; this is, however, conservative. A better curve fit is suggested by

$$
\begin{aligned}
& \text { (present) } \quad K_{\mathrm{t}}=1.8755+0.1397\left(\frac{0.1}{r / d}\right)-0.0018\left(\frac{0.1}{r / d}\right)^{2}, \\
& r / d \in[0.003: 0.07]
\end{aligned}
$$

$$
\begin{aligned}
& \text { (Pilkey) } \quad K_{\mathrm{t}}=1.9753+0.1434\left(\frac{0.1}{r / d}\right)-0.0021\left(\frac{0.1}{r / d}\right)^{2}, \\
& r / d \in[0.005: 0.07]
\end{aligned}
$$

The curve fit is given for the specific case $b / d=0.25$ and $t / d=0.125$. The curve fit from Pilkey [17] is given in equation (20). The average $K_{\mathrm{t}}$ given by equation (20) is in an average overestimation of 4 per cent relative to equation (19).

The keyway design is controlled by four variables; diameter $d$, depth $t$, width $b$, and fillet ratio $r$. For specific values it is possible to find the stress concentration factor $K_{\mathrm{t}}$ as described in section 2 . However, for easy reference it would be advantageous to have an algebraic expression for the stress concentration factor similar to the curve fit (19). Making an expression for the stress concentration that covers all the different design possibilities is not attempted here. Instead an attempt to link the
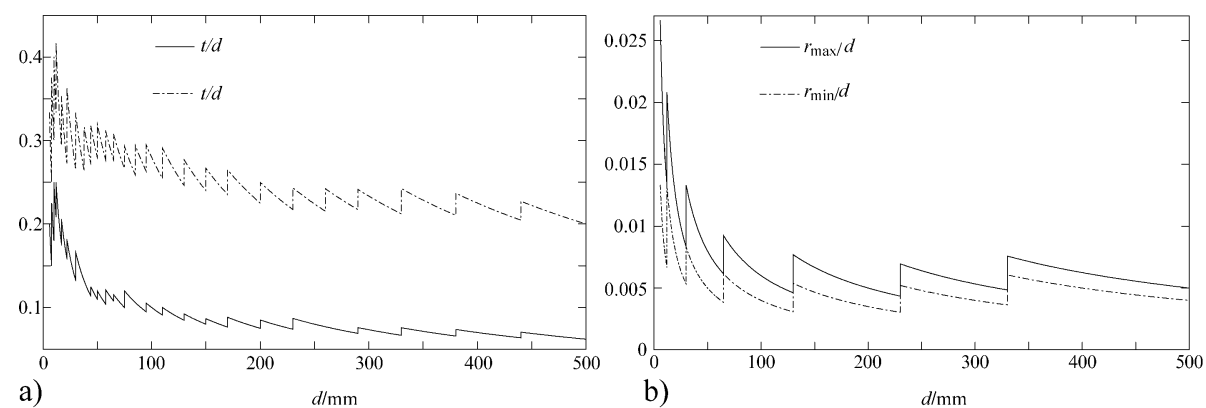

Fig. 5 (a) The depth ratio $t / d$ and width ratio $b / d$ as a function of the shaft diameter according to DIN 6885; (b) the upper and lower limits for the fillet ratio $r / d$ as a function of the shaft diameter according to DIN 6885 
design variable related to the DIN standard is performed. From a width ratio to thickness ratio plot it can be seen that these two design parameters fall naturally in two groups depending on the shaft diameter. Figure 6(a) is for the diameter range $6 \mathrm{~mm} \leqslant d \leqslant 38 \mathrm{~mm}$ and Fig. 6 (b) is for the diameter range $38 \mathrm{~mm} \leqslant d \leqslant 500 \mathrm{~mm}$. The assumption made here is that a linear curve fit to the data is appropriate; this removes one design parameter (the width $b$ ) because this is now linked to the depth $t$. The linear curve fits are

$$
\begin{aligned}
& \frac{b}{d}=1.2662 \frac{t}{d}+0.0886, \quad d \in[6: 38] \mathrm{mm} \\
& \frac{b}{d}=1.6683 \frac{t}{d}+0.1055, \quad d \in[38: 500] \mathrm{mm}
\end{aligned}
$$

It should be noted that no attempt is made for having continuity at $d=38 \mathrm{~mm}$.

Two numerical experiments have been carried out; one for the diameter range $6 \mathrm{~mm} \leqslant d \leqslant 38 \mathrm{~mm}$ shown in Fig. 7 and the other for the diameter range $38 \mathrm{~mm} \leqslant d \leqslant 500 \mathrm{~mm}$ shown in Fig. 8. In both cases the DIN norm specifies different limits to the design variables. From the numerical calculations (the points) it is clear that it is possible to make a simple curve fit that can represent the results. It should be noted that the results presented in Figs 7 and 8 are based on the design constraints specified by equations (21) and (22).

A curve fit is made for each of the two diameter ranges. It should be noted that the validity of the curve fit is bounded by the design space shown in Figs 7 and 8 respectively. The curve fits are given by
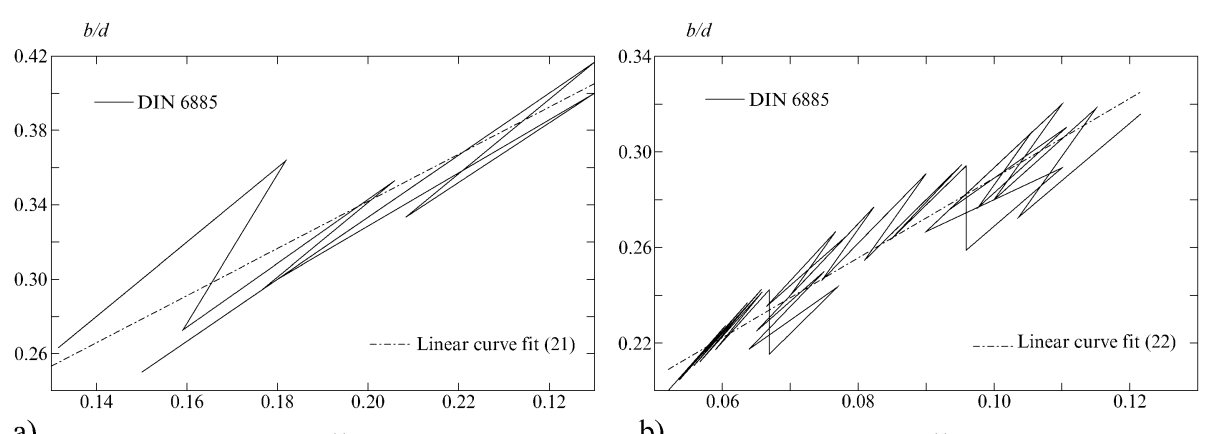

a)

b)

Fig. 6 (a) The width ratio as a function of the depth ratio for the diameter range $6 \mathrm{~mm} \leqslant d \leqslant 38 \mathrm{~mm}$ according to DIN 6885; the linear curve fit (21) is also shown. (b) the width ratio as a function of the depth ratio for the diameter range $38 \mathrm{~mm} \leqslant$ $d \leqslant 500 \mathrm{~mm}$ according to DIN 6885 ; the linear curve fit (22) is also shown

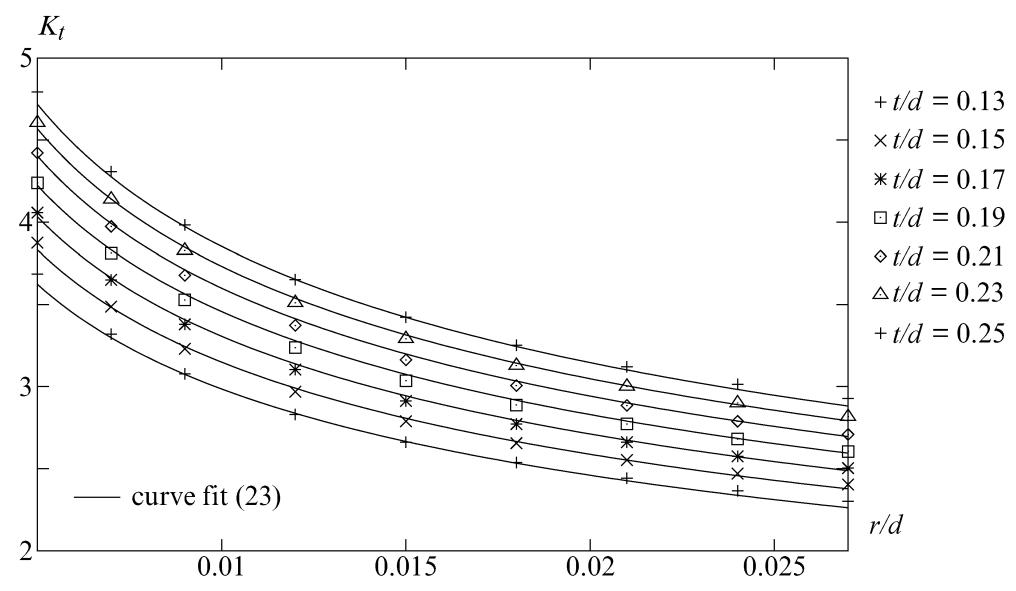

Fig. 7 The stress concentration factor as a function of the fillet ratio for diameter range $6 \mathrm{~mm} \leqslant d \leqslant 38 \mathrm{~mm}$. For this diameter range the fillet ratio fulfils $0.005 \leqslant r / d \leqslant 0.027$ and the depth ratio fulfils $0.13 \leqslant t / d \leqslant 0.25$, according to DIN 6885 . The width ratio $b / d$ is linked to the depth ratio through (21). The numerical calculations are shown by points and the full lines are the curve fit to the data, see equation (23) 


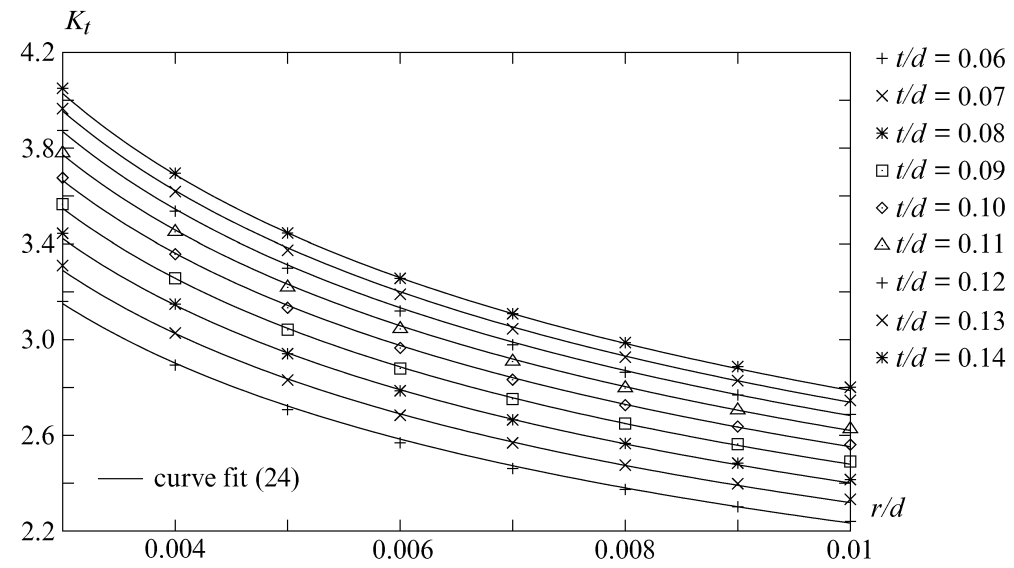

Fig. 8 The stress concentration factor as a function of the fillet ratio for the diameter range $38 \mathrm{~mm} \leqslant d \leqslant 500 \mathrm{~mm}$. For this diameter range the fillet ratio fulfils $0.003 \leqslant r / d \leqslant 0.01$ and the depth ratio fulfils $0.06 \leqslant t / d \leqslant 0.14$, according to DIN 6885 . The width ratio $b / d$ is linked to the depth ratio through equation (22). The numerical calculations are shown by points and the full lines are the curve fit to the data, see equation (24)

$$
\begin{aligned}
K_{\mathrm{t}}= & \left(1.4786 \frac{t}{d}+0.6326\right) \\
& \times\left(\frac{r}{d}\right)^{\left[0.869(t / d)^{2}-0.4392(t / d)-0.2369\right]}, \\
d \in & {[6: 38] \mathrm{mm} } \\
K_{\mathrm{t}}= & \left(1.0428 \frac{t}{d}+0.5355\right) \\
& \times\left(\frac{r}{d}\right)^{\left[2.8074(t / d)^{2}-0.8091(t / d)-2476\right]} \\
d \in & {[38: 500] \mathrm{mm} }
\end{aligned}
$$

It should be noted that no attempt is made for having continuity at $d=38 \mathrm{~mm}$.

With the two curve fits, an easy stress concentration factor estimation for the keyways prismatic part in pure torsion for designs that follow DIN 6885 is given. In the case of a specific design that does not follow the standard DIN 6885 the full numerical simulation specified in section 2 is needed. The stress concentration factor that results from a standard keyway can also be shown graphically, as in Fig. 9. The points correspond to the upper and lower limits for the fillet ratio here connected by straight lines. The $K_{\mathrm{t}}$ factor for different designs will lie in the band defined by the two lines. The top line can be used as a worst-case stress concentration factor.

\section{KEYWAY OPTIMIZATION}

In keyway design, as in many other designs within machine elements, the standard preferred shape is

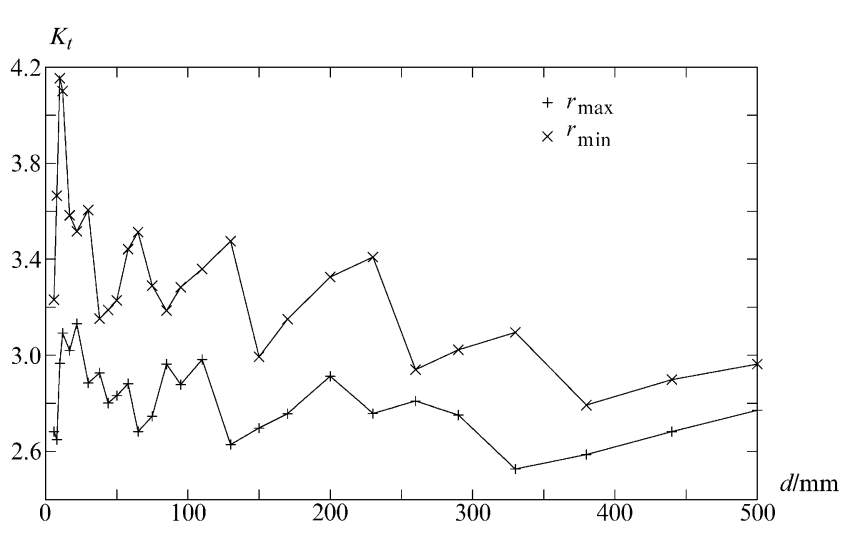

Fig. 9 The stress concentration factor for the prismatic part of a keyway in pure torsion as a function of the diameter. The different design variables are controlled by DIN 6885. The point corresponds to numerical simulations; these are connected by straight lines

the circle or a semicircle. This is probably attributable to the simple parameterization and/or ease of manufacturing. For the sled-runner design or the profile keyway there is, however, no difficulty in introducing a different fillet shape. It is well known from shape optimization that the circular shape is seldom optimal with respect to stress concentrations, see e.g. Pedersen and Pedersen [19]. In numerical shape optimization it is important to have a detailed or preferably analytical shape description. Analytical description also makes verification and comparison possible for other designs. Another reason is that it is known from shape optimization (see e.g. Ding [22] and references therein) that the FE model nodes cannot be used as design parameters. 
From a practical point of view focus should be on simplicity, although the optimization result should still be near to the optimal design. That a given parameterization is sufficiently flexible, i.e. that it can return optimal designs, can only be checked or verified after an actual optimization procedure. If the stress is constant along major parts of the surface then the shape is assumed to be optimal, see Pedersen and Pedersen [19].

The parameterization chosen here is to use the super ellipse due to the simple parameterization and owing to previous results obtained with this shape in relation to stress concentrations for other problems. The design domain is shown in Fig. 10, where the elliptical shape can be seen for the fillet.

The super ellipse (with super elliptical power $\eta$ ) is in parametric form given by

$$
\begin{aligned}
& X=L_{1}+A \cos (\alpha)^{(2 / \eta)}, \quad \alpha \in\left[0: \frac{\pi}{2}\right] \\
& Y=L_{2}+B \sin (\alpha)^{(2 / \eta)}, \quad \alpha \in\left[0: \frac{\pi}{2}\right]
\end{aligned}
$$

The keyway design is, according to Fig. 10, fully controlled by five design parameters: width $b$, depth $t$, length $L_{1}$ and $L_{2}$, and super ellipse power $\eta$. In all performed optimizations some of these parameters are assumed to be given, which leads to only two active design parameters. All parameter studies are performed for a $100 \mathrm{~mm}$ shaft and the width in all examples is chosen according to the standard, i.e. $b=28 \mathrm{~mm}$. This is of course a specific choice of shaft diameter but the results will indicate what level of stress improvements are possible more generally.

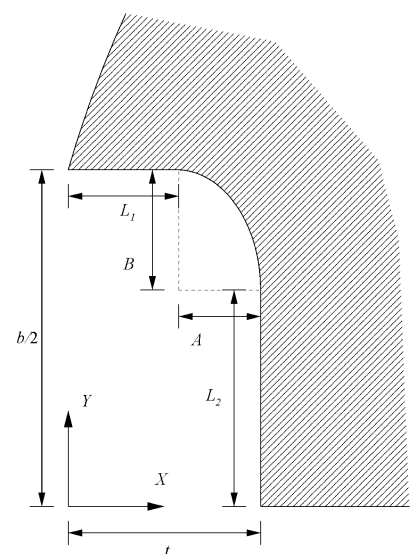

Fig. 10 The design domain: half a keyway where the fillet is a super ellipse with semi-major axes $A$ and $B$

\subsection{Design revision 1}

In the first design revision allowance is only made for the smallest possible design change relative to the original design as specified by the DIN standard. The preselected values are

$$
b=28 \mathrm{~mm}, \quad t=8 \mathrm{~mm}, \quad L_{1}=7.4 \mathrm{~mm}
$$

i.e. the width and depth comply with the standard and the shoulder length $L_{1}$ complies with the largest allowable fillet ratio $r=0.6 \mathrm{~mm}$. The design variables here are therefore the bottom length $L_{2}$ and the super elliptical power $\eta$. The parameter study results in optimized values

$$
L_{2}=13.19 \mathrm{~mm}, \quad \eta=1.63
$$

An iso line plot of the largest principal stress is presented in Fig. 11. From this figure it can be seen that the iso lines close to the fillet run parallel, indicating constant stress along the shape. This is visualized in Fig. 12, which shows the stress concentration factor along the keyway boundary. From the close up in Fig. 12(b) it is seen that the stress is close to being constant along the fillet. The optimized stress concentration factor value is $K_{\mathrm{t}}=2.53$ and this number can be compared to the previous found result of $K_{\mathrm{t}}=2.93$ for the DIN standard with the semicircular fillet design. The maximum stress has therefore been reduced with 13.6 per cent with this rather small design change. Owing to the unchanged shoulder length, $L_{1}$, this keyway design can be assumed to function exactly as the original design.

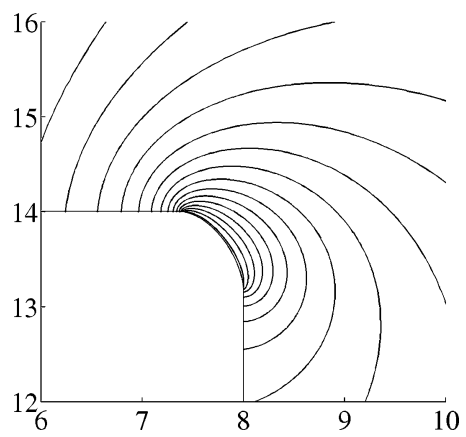

Fig. 11 Iso lines of largest principal stress for the optimized design

\subsection{Design revision 2}

In the second design revision the constraint on the depth $t$ is removed, allowing a deeper keyway to 


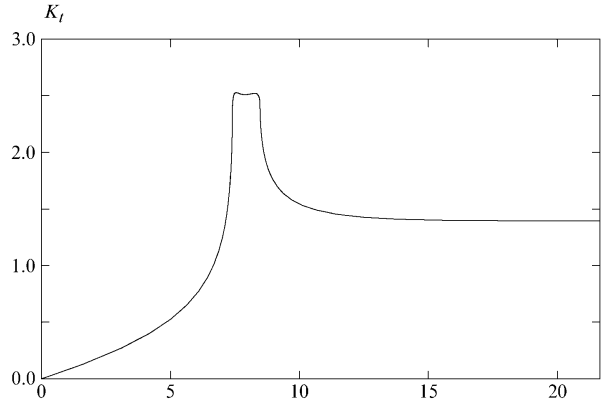

a)

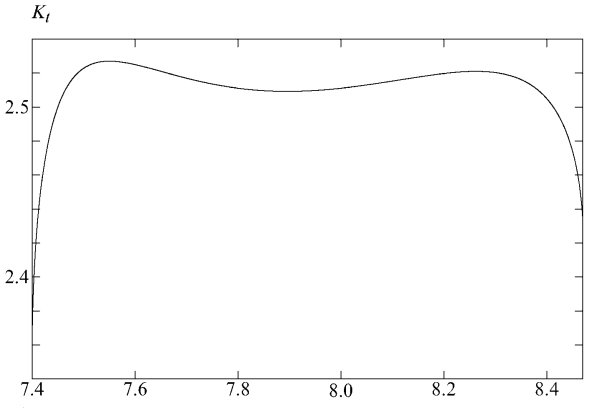

b)

Fig. 12 The figures are for half the keyway and show the stress concentration as a function of the arc length: (a) the stress concentration factor along the keyway boundary starting from the external point until the centre point; (b) stress concentration close up, here only shown along the super ellipse; the maximum stress concentration factor is $K_{\mathrm{t}}=2.53$

investigate the possible stress improvements that can be achieved by this. The remaining preselected values are

$$
b=28 \mathrm{~mm}, \quad L_{1}=7.4 \mathrm{~mm}
$$

i.e. the width complies with DIN 6885 and the shoulder length $L_{1}$ complies with the largest allowable fillet ratio $r=0.6 \mathrm{~mm}$. In principal there are now three design variables: the depth $t$ the length $\mathrm{L}_{2}$ and the super elliptical power $\eta$. However, from the preformed parameter study it is found that $L_{2}=0$ and the length parameter $L_{2}$ is not an active design parameter. The parameter study results in the optimized values

$$
L_{2}=0 \mathrm{~mm}, \quad t=11.51, \quad \eta=1.99
$$

An iso line plot of largest principal stress is presented in Fig. 13. The iso lines in this figure close to the fillet are, as in the previous example, parallel to the fillet, indicating constant stress along the shape. This is illustrated in Fig. 14, which shows the stress concentration factor along the keyway boundary. The dotted straight line indicates the maximum value, which in this case is $K_{\mathrm{t}}=1.65$. It is seen that the stress is close to being constant along the fillet. The plot shows the stress concentration factor along half the keyway. It is known that at the starting corner the stress must be zero and then the stress must build up to the maximum value, which in this case is almost constant along the fillet. Although the parameterization chosen is very simple with only two active design parameters, the design is close to the optimum. A better parameterization with more design variables might lead to a more constant stress along the shape, but from Fig. 14 it is seen that the scope for improvement is small.

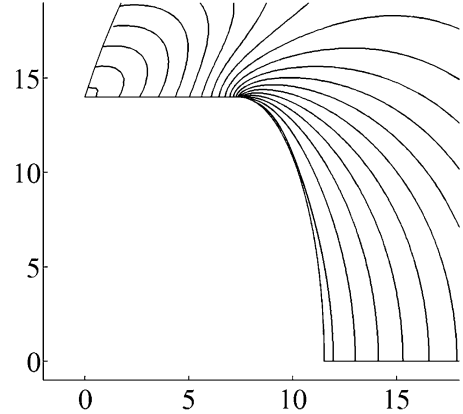

Fig. 13 Iso lines of largest principal stress for the optimized design

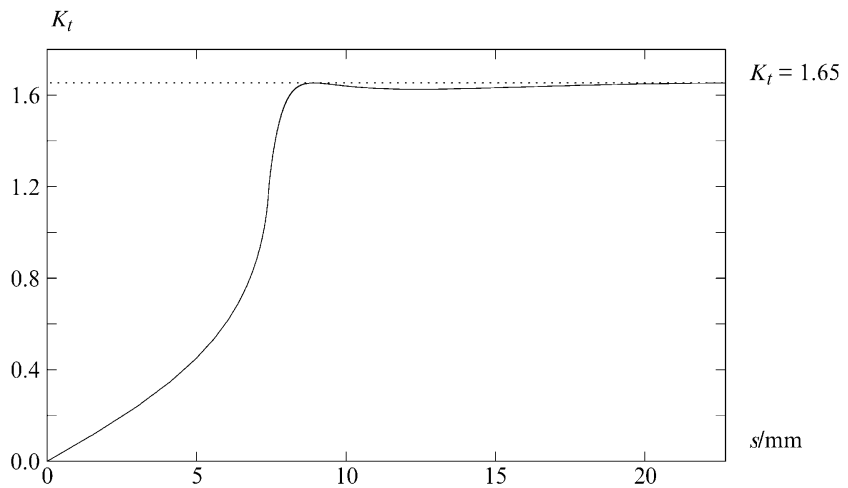

Fig. 14 The stress concentration as a function of the arc length along the keyway from the external point up to the centre point

The maximum stress for this design has been reduced by 43.7 per cent relative to the original design. The design improvement has been achieved using the same shoulder length as specified by the standard. The load-carrying capacity is therefore identical. The key design must, however, be changed to comply with this new keyway design. 


\subsection{Design revision 3}

In the final revision the only fixed variables are the width and the depth

$$
b=28 \mathrm{~mm}, \quad t=8 \mathrm{~mm}
$$

In this example there are three design variables but as was the case in the previous example the result of a parameter study is that the length $L_{2}$ should be zero. The optimized design variables are

$$
L_{2}=0 \mathrm{~mm}, \quad L_{1}=4.56, \quad \eta=2.22
$$

The design and an iso line plot of largest principal stress are presented in Fig. 15.

The comments are all identical with the previous paragraph. The stress concentration in this case improved even more. The dotted straight line indicates the maximum value, which in this case is $K_{\mathrm{t}}=1.50$. This is a 48.8 per cent reduction in the maximum stress. The improvement here relative to the previous example is partly due to the smaller keyway. The load-carrying capacity with respect to bearing failure is in this case smaller relative to the previous example owing to the smaller depth.

\section{SUGGESTED NEW STANDARD}

The examples in the previous section have shown the potential stress reduction from different design modifications to the standard keyway design. The results indicate that to utilize the stress reduction fully the design must be customized for the different shaft diameters. There are, however, also relative large improvements for designs that are slightly modified compared to the optimal. The suggested new standard follows the design revision 2. Because of differences in the original design it is suggested to have a small difference for shaft diameters smaller

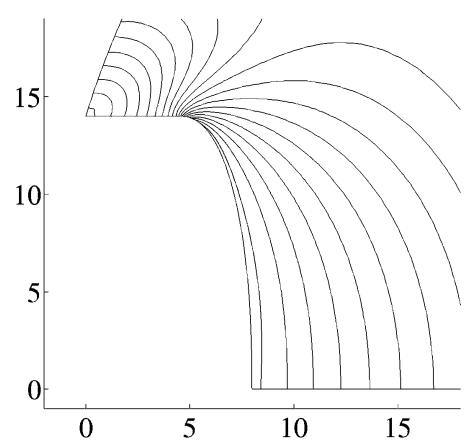

Fig. 15 Iso lines of largest principal stress for the optimized design than or greater than $d=38 \mathrm{~mm}$. The common design variables for the new suggested keyway design are

(a) $L_{1}=$ minimum allowable shoulder length according to DIN 6885;

(b) $L_{2}=0$;

(c) $\eta=2$;

(d) $b=$ DIN 6885 standard;

(e) $t=1.4 L_{1}$ for $6 \mathrm{~mm} \leqslant d \leqslant 38 \mathrm{~mm}$ and $t=1.5 L_{1}$ for $38 \mathrm{~mm} \leqslant d \leqslant 500 \mathrm{~mm}$.

The $K_{\mathrm{t}}$ factors for the diameter range $6 \mathrm{~mm} \leqslant$ $d \leqslant 500 \mathrm{~mm}$ are shown in Fig. 17 together with the minimum obtainable stress concentration using the standard. It can be seen that for most of the diameter range the $K_{\mathrm{t}}$ factor is almost constant. The design is best for the larger diameter range but always better than that given by the standard. The smallest difference is achieved for $d=8 \mathrm{~mm}$ where the minimum stress concentration specified by DIN 6885 is $K_{\mathrm{t}}=2.65$ where the new keyway design has $K_{\mathrm{t}}=2.41$, i.e. a 9 per cent reduction in the stress. For most of the diameter range the improvement is much larger with a reduction in the maximum stress of about 35 per cent. This number should be compared to the 43.7 per cent improvement reported in the previous section.

\section{CONCLUSION}

This paper has demonstrated how it is rather simple to find the stress concentration factors for the prismatic part of a keyway in pure torsion. Using the keyway design as defined by DIN 6885 the result of the paper is a simple algebraic expression for the stress concentration factor. Also presented is a band within which the stress concentration factors for the DIN 6885 lies.

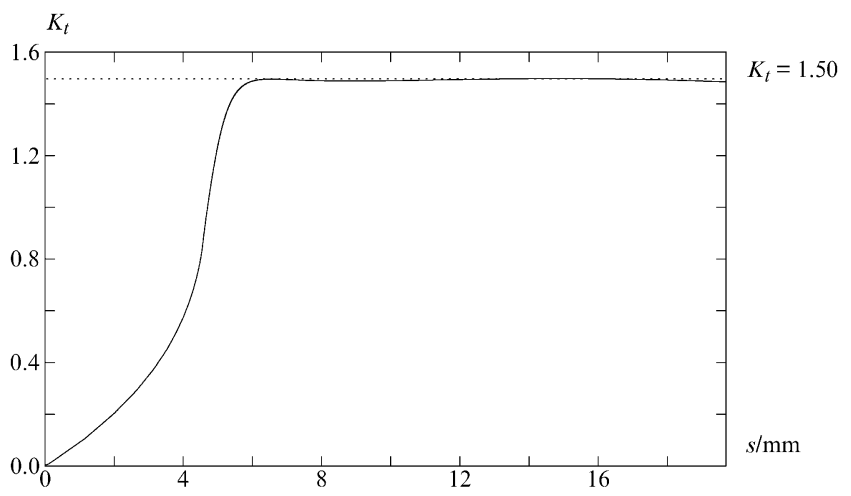

Fig. 16 The stress concentration as a function of the arc length along the keyway from the external point up to the centre point 


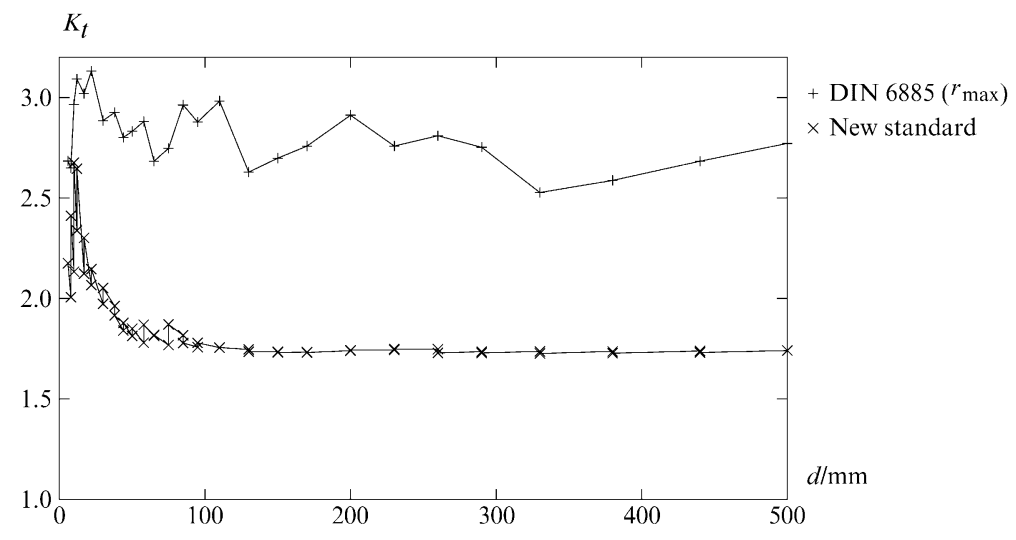

Fig. 17 The stress concentration factor for the prismatic part of a keyway in pure torsion as a function of the diameter. The top curve is for the DIN 6885 design with the maximum allowable fillet radius. The bottom curve is for the new proposed keyway design. The point corresponds to numerical simulations, which are connected by straight lines

The second part of the paper is concerned with a keyway design revision for minimizing the stress concentration factor. Three different revisions to the standard design are shown. The reported stress level lowering is significant with up to almost a 50 per cent reduction. This is achieved by a rather simple shape modification by introducing the super ellipse and using only two design parameters. The optimized stress concentrations are found through a pure torsional loading based on the result in reference [15]. Loading the keyway through a key will lead to a variation of the obtained stress concentration factors.

Finally an overall design revision or new standard keyway design for the whole diameter range is proposed. Resulting in, on average, a 35 per cent reduction in the maximum stress relative to the best design that can be achieved by following the DIN 6885. The smallest improvement reported is 9 per cent but this is the price to pay for choosing a new standard relative to a customized design for each shaft diameter.

\section{ACKNOWLEDGEMENT}

The author would like to thank Professor Pauli Pedersen and Professor Peder Klit for their discussions and suggestions.

(c) Author 2010

\section{REFERENCES}

1 Deutsches Institut für Normung. DIN 6885-1. Paßfedern nuten, 1968 (DIN, Berlin), in German.
2 Norton, R. L. Machine design: an integrated Approach, third edition, 2006 (Pearson Education, Upper Saddle River, NJ).

3 Shigley, J. E. and Michke, C. R. Mechanical engineering design, sixth edition, 2003 (McGraw Hill, Singapore).

4 Orthwein, W. C. A new key and keyway design. J. Mech Des. Trans. ASME, 1979, 101(2), 338-341.

5 Merritt, H. E. The design of cylindrical keys. Machinery (Lond.), 1926, 27(701), 729-732.

6 Kuske, A. Erhöhung der Lebensdauer durch Verbesserung der Bauteilgestalt. Stahl und Eisen, 1971, $91(8), 446-51$.

7 Filon, L. N. G. On the resistance to torsion of certain forms of shafting, with special reference to the effect of keyways. Phil. Trans. Roy. Soc. Lond. Ser. A, Containing Papers of a Mathematical or Physical Character, 1900, 193, 309-352.

8 Leven, M. M. Stresses in keyways by photoelastic methods and comparison with numerical solutions. Proc. Soc. Experl Stress Analysis, 1949, 7(2), 141-154.

9 Fessler, H., Rogers, C. C., and Stanley, P. Stresses at end-milled keyways in plain shafts subjected to tension, bending, and torsion. J. Strain Analysis, 1969, 4(3), 180-189.

10 Fessler, H., Rogers, C. C., and Stanley, P. Stresses at keyway ends near shoulders. J. Strain Analysis, 1969, 4(4), 267-277.

11 Orthwein, W. C. Keyway stresses when torsional loading is applied by the keys. Expl. Mechanics, 1975, 15(6), 245-248.

12 Eissa, M. and Fessler, H. Reduction of elastic stress concentrations in end-milled keyed connections. Expl Mechanics, 1983, 23(4), 401-408.

13 Fessler, H. and Appavoo, T. On the effect of key edge shape on keyway edge stresses in shafts in torsion. $J$. Strain Analysis Engng Des., 1989, 24(3), 121-125.

14 Terada, K. Erneute Untersuchung der Formzahl für Keilnuten. Materialprüfung, 1963, 5(10), 385-387.

15 Okubo, H., Hosono, K., and Sakaki, K. The stress concentration in keyways when torque is trans- 
mitted through keys. Expl Mechanics, 1968, 8(8), J 375-380.

16 Peterson, R. E. Stress concentration design factors, 1953 (John Wiley, New York).

17 Pilkey, W. D. Peterson's stress concentration factors, 1997 (John Wiley, New York).

18 Peterson, R. E. Fatigue of shafts having keyways. ASTM Proc., 1932, 32(part 2), 413-419.

19 Pedersen, N. L. and Pedersen, P. Design of notches and grooves by means of elliptical shapes. J. Strain Analysis Engng Des., 2008, 43(1), 1-14.

20 Pedersen, N. L. Reducing bending stress in external spur gears by redesign of the standard cutting tool. Struct. Multidisciplinary Optimization, 2009, 38(3), 215-227.

21 COMSOL AB. Stockholm. see www.comsol.se.

22 Ding, Y. Shape optimization of structures: a literature survey. Computers and Struct, 1986, 24(6), 985-1004.

\section{APPENDIX}

\section{Notation}

$b$

C

$d$

width of keyway

constant

diameter of shaft

G
$K_{\mathrm{t}}$

$K_{\mathrm{ts}}$

$l$

$L_{1}, L_{2}$

$M_{\mathrm{t}}$

$n$

$r$

$s$

$t$

$v$

$\eta$

$\theta$

$\sigma$

$\sigma_{\max }$

$\sigma_{\text {nom }}$

$\tau$

$\tau_{\max }$

$\tau_{\text {nom }}$

$\phi$

$\Psi$ torsional stiffness factor of

cross-section

theoretical stress concentration

factor (normal stress)

theoretical stress concentration factor (shear stress)

length of shaft

design parameters

torsional moment

normal vector component

fillet radius

arc length

depth of keyway

displacement

super elliptical power (design parameter)

angular rotation per length

normal stress

maximum stress

nominal stress

shear stress

maximum shear stress

nominal shear stress

angular rotation of torsional

cross-section

warping function 